\title{
Stability and Oscillatory Behavior of the Solutions on a Class of Coupled Van der Pol-Duffing Equations with Delays
}

\author{
Chunhua Feng \\ Department of Mathematics and Computer Science, Faculty of Alabama State University, Montgomery, USA
}

Email address:

cfeng@alasu.edu

\section{To cite this article:}

Chunhua Feng. Stability and Oscillatory Behavior of the Solutions on a Class of Coupled Van der Pol-Duffing Equations with Delays. Mathematics and Computer Science. Vol. 4, No. 6, 2019, pp. 104-111. doi: 10.11648/j.mcs.20190406.12

Received: October 31, 2019; Accepted: November 29, 2019; Published: December 10, 2019

\begin{abstract}
In the present paper, a class of coupled van der Pol-Duffing oscillators with a nonlinear friction of higher polynomial order model which involves time delays is investigated. The coefficients of the highest order of the polynomial determine the boundedness of the solutions. With special attention to the boundedness of the solutions and the instability of the unique equilibrium point of linearized system, some sufficient conditions to guarantee the existence of oscillatory solutions for the model are obtained based on the generalized Chafee's criterion. Convergence of the trivial solution is determined by the negative real part of eigenvalues of the linearized system. Examples are provided to demonstrate the reduced conservativeness for the parameters of the proposed results. The results obtained shown that the passive decay rate in the model affects the oscillatory frequency and amplitude. When a permanent oscillation occurred, time delays affect mainly oscillatory frequency and amplitude slightly.
\end{abstract}

Keywords: Coupled Van der Pol-Duffing Equation, Delay, Stability, Oscillation

\section{Introduction}

It is known that the van der Pol (VDP) oscillator could model the typical self-excited or self-sustained oscillation. Various coupled van der Pol or van der Pol-Duffing equations have been applied in physics and engineering. Many good results have appeared [1-12]. For the following three-dimensional autonomous van der Pol-Duffing type oscillator system:

$$
\left\{\begin{array}{c}
x^{\prime}(t)=y(t), \\
y^{\prime}(t)=-x(t)+\beta x^{3}(t)+\varepsilon\left(1-x^{2}(t)\right) y(t)-k z(t),(1) \\
z^{\prime}(t)=y(t)-z(t) .
\end{array}\right.
$$

By analyzing the stability of the equilibrium points, the existence of Hopf bifurcation is established [1]. Barron has considered the stability of a ring of coupled van der Pol oscillators with non-uniform distribution of the coupling parameter as follows:

$$
\begin{gathered}
x_{i}^{\prime \prime}(t)+a\left(x_{i}^{2}(t)-1\right) x_{i}^{\prime}(t)+x_{i}(t)=b_{i}\left(x_{i-1}(t)-\right. \\
\left.2 x_{i}(t)+x_{i+1}(t)\right)
\end{gathered}
$$

where $1 \leq i \leq n, b_{i}$ are the coupling parameter corresponding to the $i$ th oscillator. For a modified hybrid van der Pol-Duffing-Rayleigh oscillator for modeling the lateral walking force on a rigid floor:

$$
\begin{gathered}
x^{\prime \prime}(t)+\gamma x(t)+w_{0}^{2} x(t)-a x^{2}(t)-b x(t) x^{\prime}(t)+ \\
c x^{3}(t)+d x^{2}(t) x^{\prime}(t)=0
\end{gathered}
$$

Kumar et al. have studied the stability of the equation (3) by the perturbation and energy balance method [3]. Rompala et al. have considered a system of three van der Pol oscillators [5]. For a ring of four mutually coupled biological systems described by coupled van der Pol oscillators, the stability boundaries and the main dynamical states have been considered on the stability maps by Kadji et al. [6]. A driven van der Pol-like oscillator with a nonlinear friction of higher polynomial order model as follows:

$$
\begin{gathered}
x^{\prime \prime}(t)-\mu\left(1-x^{2}(t)+\alpha x^{4}(t)-\beta x^{6}(t)\right) x^{\prime}(t)+x(t)= \\
\theta_{0} \sin \omega t
\end{gathered}
$$

The effects of noise correlation on the coherence of a forced van der Pol type birhythmic system has been 
investigated by Yamapi et al. [7]. It is well known that the time delay is inevitable in many physical and biological phenomena such as manufacturing process, nuclear reactors, rocket motors, mechanical controlling systems, population dynamics, and so on. Naturally the time delay coupled van der Pol equations also have been extensively studied by many researchers [13-24]. For example, Li et al. have studied the coupled van der Pol oscillators with two kinds of delays [13]:

$$
\left\{\begin{array}{l}
y_{1}^{\prime \prime}(t)+w_{1}^{2} y_{1}(t)-\varepsilon\left(1-y_{1}^{2}(t)\right) y_{1}^{\prime}(t)=\varepsilon \alpha\left(y_{2}(t-\tau)+y_{2}^{\prime}(t-\tau)\right), \\
y_{2}^{\prime \prime}(t)+w_{2}^{2} y_{2}(t)-\varepsilon\left(1-y_{2}^{2}(t)\right) y_{2}^{\prime}(t)=\varepsilon \alpha\left(y_{1}(t-\tau)+y_{1}^{\prime}(t-\tau)\right) .
\end{array}\right.
$$

Zhang and $\mathrm{Gu}$ used the theory of normal form and central manifold theorem to discuss the following time delay system [14]:

$$
\left\{\begin{array}{l}
x_{1}^{\prime \prime}(t)+\varepsilon\left(x_{1}^{2}(t)-1\right) x_{1}^{\prime}(t)+x_{1}(t)=\alpha\left(y_{1}(t-\tau)-x_{1}(t)\right), \\
y_{1}^{\prime \prime}(t)+\varepsilon\left(y_{1}^{2}(t)-1\right) y_{1}^{\prime}(t)+y_{1}(t)=\alpha\left(x_{1}(t-\tau)-y_{1}(t)\right) .
\end{array}\right.
$$

Motivated by the above models, in this paper we consider the following a ring of time delays Duffing-van der Pol-like oscillator with a nonlinear friction of higher polynomial order system:

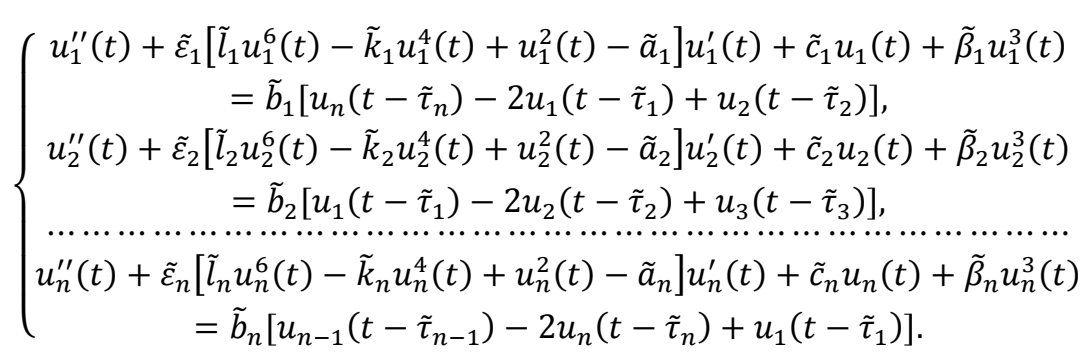

where $0<\tilde{l}_{i}, \tilde{k}_{i}$ and $0<\tilde{\varepsilon}_{i} \ll 1 ; \tilde{c}_{i}, \tilde{\beta}_{i}, \tilde{a}_{i}, \tilde{b}_{i} \in \mathrm{R}$ for each $i=1,2, \ldots, n, 0 \leq \tilde{\tau}_{i}$ are time delays. Our aim is to investigate the dynamical behavior of $n$ coupled oscillators by means of the generalized Chafee's criterion [25, 26].

\section{Preliminaries}

For convenience, setting $\tilde{\varepsilon}_{i}=\varepsilon_{2 i}, \tilde{l}_{i}=l_{2 i}, \tilde{k}_{i}=k_{2 i}, \tilde{a}_{i}=a_{2 i}, \tilde{c}_{i}=c_{2 i-1}, \tilde{b}_{i}=b_{2 i-1}, \tilde{\beta}_{i}=\beta_{2 i-1}, \tilde{\tau}_{i}=\tau_{2 i-1}(1 \leq i \leq n)$. Then the coupled system (7) can be written as the following equivalent system:

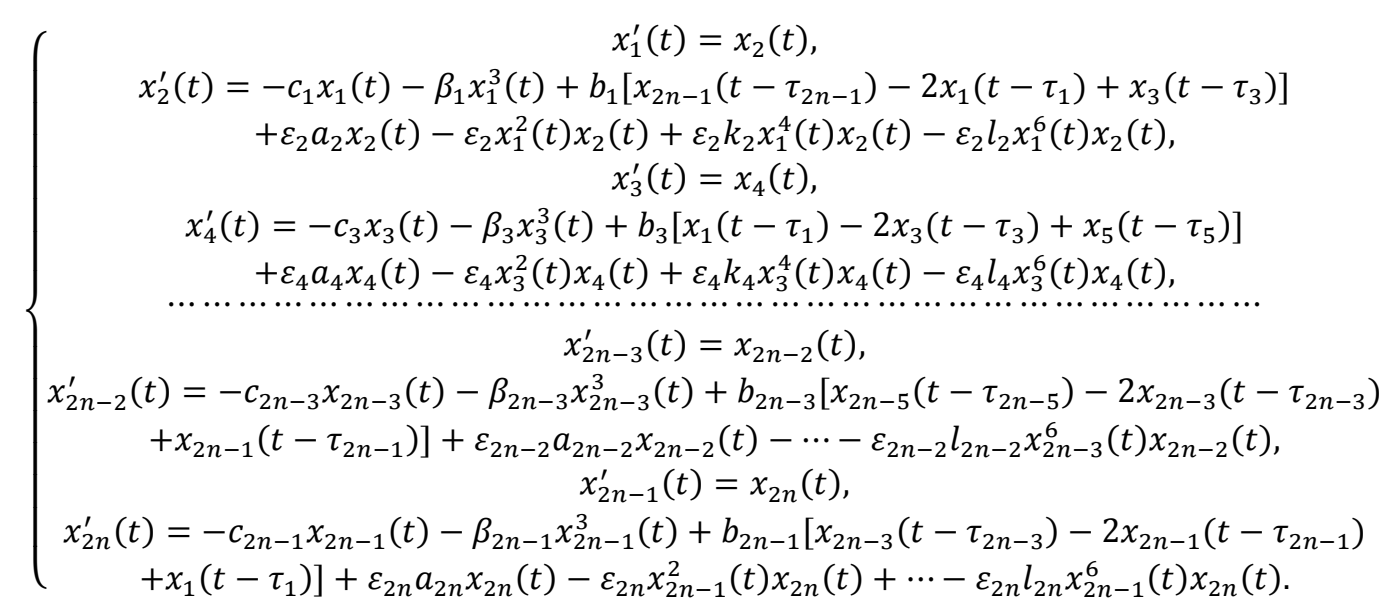

The matrix form of system (8) is the following:

$$
X^{\prime}(t)=A X(\mathrm{t})+B X(t-\tau)+g(X)
$$

where $X(t)=\left[x_{1}(t), x_{1}(t), \cdots, x_{2 n}(t)\right]^{T}$,

$$
X(t-\tau)=\left[x_{1}\left(t-\tau_{1}\right), 0, x_{3}\left(t-\tau_{1}\right), \cdots, x_{2 n-1}(t\right.
$$$$
\left.\left.-\tau_{2 n-1}\right), 0\right]^{T} \text {, }
$$

$g(X)=\left[0,-\beta_{1} x_{1}^{3}(t)-\varepsilon_{2} x_{1}^{2}(t) x_{2}(t)+\varepsilon_{2} k_{2} x_{1}^{4}(t) x_{2}(t)-\right.$ $\varepsilon_{2} l_{2} x_{1}^{6}(t) x_{2}(t), 0, \cdots, 0,-\beta_{2 n-1} x_{2 n-1}^{3}(t)-$ $\varepsilon_{2 n} x_{2 n-1}^{2}(t) x_{2 n}(t)+\varepsilon_{2 n} k_{2} x_{2 n-1}^{4}(t) x_{2 n}(t)-$ $\left.\varepsilon_{2 n} l_{2 n} x_{2 n-1}^{6}(t) x_{2 n}(t)\right]^{T}$. Both $A=\left(a_{i j}\right)_{2 n \times 2 n}$ and $B=$ $\left(b_{i j}\right)_{2 n \times 2 n}$ are $2 n$ by $2 n$ matrices as follows:

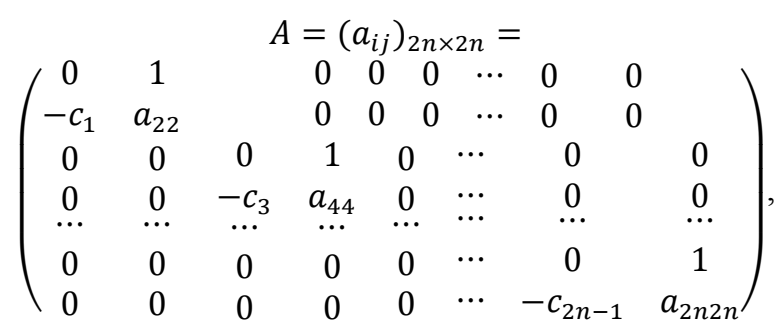


where $a_{22}=\varepsilon_{2} a_{2}, a_{44}=\varepsilon_{4} a_{4}, a_{2 n 2 n}=\varepsilon_{2 n} a_{2 n}$.

$$
=\left(\begin{array}{cccccccccc}
0 & 0 & \multicolumn{8}{c}{B=\left(b_{i j}\right)_{2 n \times 2 n}} \\
-2 b_{1} & 0 & \multicolumn{1}{c}{b_{1}} & 0 & 0 & \cdots & 0 & 0 & \\
0 & 0 & 0 & 0 & 0 & \cdots & b_{1} & 0 & \\
b_{3} & 0 & -2 b_{3} & 0 & 0 & \cdots & 0 & 0 \\
\cdots & \cdots & \cdots & \cdots & \cdots & \cdots & & 0 & 0 \\
0 & 0 & 0 & 0 & 0 & \cdots & 0 & 0 \\
b_{2 n-1} & 0 & 0 & 0 & 0 & \cdots & -2 b_{2 n-1} & 0
\end{array}\right) .
$$

Obviously, the origin $x_{k}=0(k=1,2, \cdots, 2 n)$ is an equilibrium of system (8). The linearization of system (9) at origin is

$$
\begin{aligned}
& X^{\prime}(t)=A X(\mathrm{t})+B X(t-\tau)
\end{aligned}
$$

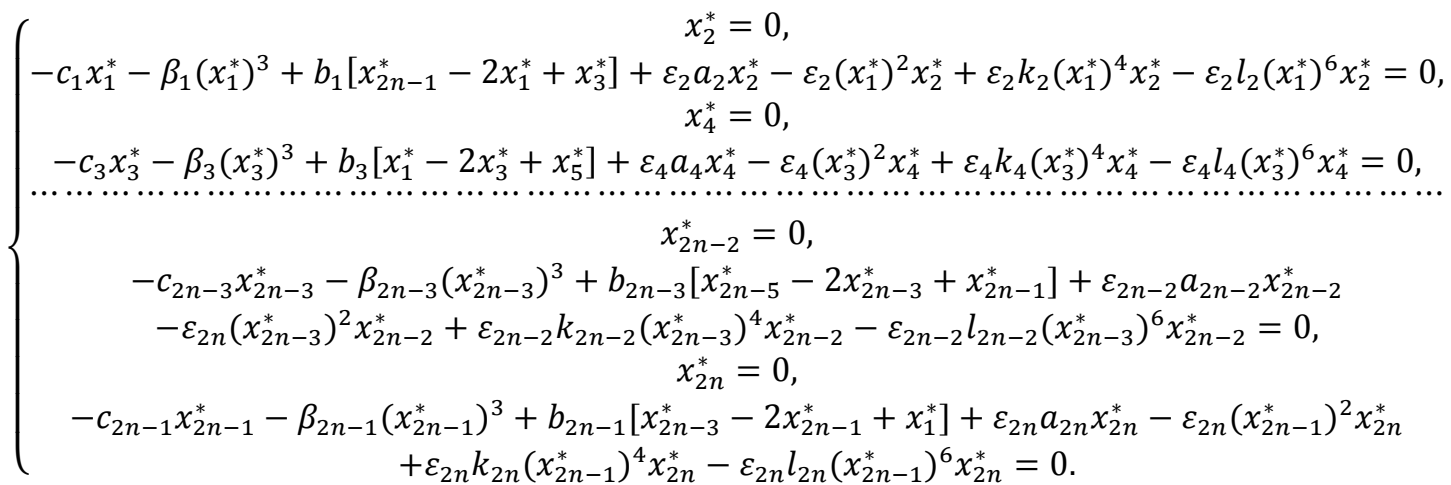

Let

$$
C=\left(c_{i j}\right)_{n \times n}=\left(\begin{array}{ccccccc}
c_{11} & 0 & b_{1} & 0 & \cdots & 0 & b_{1} \\
b_{3} & 0 & c_{23} & 0 & \cdots & 0 & 0 \\
\cdots & \cdots & \cdots & \cdots & \cdots & \cdots & \cdots \\
0 & 0 & 0 & 0 & \cdots & 0 & b_{2 n-3} \\
b_{2 n-1} & 0 & 0 & 0 & \cdots & 0 & c_{n n}
\end{array}\right) \text {, }
$$

where $\quad c_{11}=-2 b_{1}-c_{1}, \quad c_{23}=-2 b_{3}-c_{3}, \cdots, \quad c_{n n}=$ $-2 b_{2 n-1}-c_{2 n-1}$.

Lemma 1 Assume that the matrix $C$ is a nonsingular matrix, then system (8) (or (9)) has a unique equilibrium.

Proof An equilibrium point $x^{*}=\left[x_{1}^{*}, x_{2}^{*}, \cdots, x_{2 n}^{*}\right]^{T}$ of system (8) is a constant solution of the following algebraic equation

Since $x_{2 i}^{*}=0(1 \leq i \leq n)$, from (11) we get

$$
\left\{\begin{array}{c}
-c_{1} x_{1}^{*}-\beta_{1}\left(x_{1}^{*}\right)^{3}+b_{1}\left[x_{2 n-1}^{*}-2 x_{1}^{*}+x_{3}^{*}\right]=0, \\
-c_{3} x_{3}^{*}-\beta_{3}\left(x_{3}^{*}\right)^{3}+b_{3}\left[x_{1}^{*}-2 x_{3}^{*}+x_{5}^{*}\right]=0, \\
\cdots \cdots \cdots \cdots \cdots \cdots \cdots \cdots \cdots \cdots \cdots \cdots \cdots \cdots \cdots \cdots \cdots \cdots \cdots \cdots \\
-c_{2 n-3} x_{2 n-3}^{*}-\beta_{2 n-3}\left(x_{2 n-3}^{*}\right)^{3}+b_{2 n-3}\left[x_{2 n-5}^{*}-2 x_{2 n-3}^{*}+x_{2 n-1}^{*}\right]=0, \\
-c_{2 n-1} x_{2 n-1}^{*}-\beta_{2 n-1}\left(x_{2 n-1}^{*}\right)^{3}+b_{2 n-1}\left[x_{2 n-3}^{*}-2 x_{2 n-1}^{*}+x_{1}^{*}\right]=0 .
\end{array}\right.
$$

System (12) can be written as a matrix form as the have following:

$$
D X^{*}=0
$$

where $X^{*}=\left[x_{1}^{*}, x_{3}^{*}, \cdots, x_{2 n-1}^{*}\right]^{T}$, and

$$
D=\left(d_{i j}\right)_{n \times n}=\left(\begin{array}{ccccc}
d_{11} & b_{1} & 0 & \cdots & b_{1} \\
b_{3} & d_{22} & b_{3} & \cdots & 0 \\
\cdots & \cdots & \cdots & \cdots & \cdots \\
0 & 0 & 0 & \cdots & b_{2 n-3} \\
b_{2 n-1} & 0 & 0 & \cdots & d_{n n}
\end{array}\right)
$$

where $\quad d_{i i}=-2 b_{2 i-1}-c_{2 i-1}-\beta_{2 i-1}\left(x_{2 i-1}^{*}\right)^{2}(1 \leq i \leq n)$. According to standard results in linear algebraic, if $D$ is a nonsingular matrix, system (13) has only one solution, namely, the trivial solution. When $x_{2 i-1}^{*}=0((1 \leq i \leq n)$, matrix $D$ changes to $C$. The proof is completed.

Lemma 2 All solutions of system (8) are uniformly bounded.

Proof Construct a Lyapunov function $V(t)=\sum_{i=1}^{2 n} \frac{1}{2} x_{i}^{2}(t)$. Calculating the derivative of $V(t)$ through system (8) we

$$
\begin{gathered}
\left.V^{\prime}(t)\right|_{(8)}=\sum_{i=1}^{2 n} x_{i}(t) x_{i}^{\prime}(t) \\
=x_{1}(t) x_{2}(t)+x_{2}(t)\left\{-c_{1} x_{1}(t)-\beta_{1} x_{1}^{3}(t)\right. \\
+b_{1}\left[x_{2 n-1}\left(t-\tau_{2 n-1}\right)-2 x_{1} t-\tau_{1}\right) \\
\left.+x_{3}\left(t-\tau_{3}\right)\right]+\varepsilon_{2} a_{2} x_{2}(t) \\
\left.-\varepsilon_{2} x_{1}^{2}(t) x_{2}(t)+\varepsilon_{2} k_{2} x_{1}^{4}(t) x_{2}(t)-\varepsilon_{2} l_{2} x_{1}^{6}(t) x_{2}(t)\right\} \\
+\cdots \cdots+x_{2 n-1}(t) x_{2 n}(t) \\
+x_{2 n}(t)\left\{-c_{2 n-1} x_{2 n-1}(t)-\beta_{2 n-1} x_{2 n-1}^{3}(t)\right. \\
+b_{2 n-1}\left[x_{2 n-3}\left(t-\tau_{2 n-3}\right) 2 x_{2 n-1}(t\right. \\
\left.\left.-\tau_{2 n-1}\right)+x_{1}\left(t-\tau_{1}\right)\right] \\
+\varepsilon_{2 n} \\
a_{2 n} x_{2 n}(t)-\varepsilon_{2 n} x_{2 n-1}^{2}(t) x_{2 n}(t) \\
+\varepsilon_{2 n} k_{2 n} x_{2 n-1}^{4}(t) x_{2 n}(t) \\
\left.-\varepsilon_{2 n} l_{2 n} x_{2 n-1}^{6}(t) x_{2 n}(t)\right\} \\
x_{1}(t)-\beta_{1} x_{1}^{3}(t) x_{2}(\mathrm{t})+b_{1} x_{2}(\mathrm{t})\left[x_{2 n-1}(t-\right. \\
\left.\left.\tau_{2 n-1}\right)-2 x_{1} t-\tau_{1}\right)+
\end{gathered}
$$




$$
\begin{gathered}
\left.x_{3}\left(t-\tau_{3}\right)\right]+\varepsilon_{2} a_{2} x_{2}^{2}(t)-\varepsilon_{2} x_{1}^{2}(t) x_{2}^{2}(t)+\varepsilon_{2} k_{2} x_{1}^{4}(t) x_{2}^{2}(t) \\
+\cdots \cdots \\
+\left(1-c_{2 n-1}\right) x_{2 n-1}(t) x_{2 n}(\mathrm{t})-\beta_{2 n-1} x_{2 n-1}^{3}(t) x_{2 n}(\mathrm{t}) \\
+b_{2 n-1} x_{2 n}(\mathrm{t}) \\
{\left[x_{2 n-3}\left(t-\tau_{2 n-3}\right)-2 x_{2 n-1}\left(t-\tau_{2 n-1}\right)+x_{1}\left(t-\tau_{1}\right)\right]} \\
+\varepsilon_{2 n} a_{2 n} x_{2 n}^{2}(t)-\varepsilon_{2 n} x_{2 n-1}^{2}(t) x_{2 n}^{2}(t) \\
+\varepsilon_{2 n} k_{2 n} x_{2 n-1}^{4}(t) x_{2 n}^{2}(t) \\
-\varepsilon_{2} l_{2} x_{1}^{6}(t) x_{2}^{2}(t)-\varepsilon_{4} l_{4} x_{3}^{6}(t) x_{4}^{2}(t)-\cdots \cdots- \\
\varepsilon_{2 n} l_{2 n} x_{2 n-1}^{6}(t) x_{2 n}^{2}(t)
\end{gathered}
$$

Obviously, $x_{1}^{6}(t) x_{2}^{2}(t), x_{3}^{6}(t) x_{4}^{2}(t), \cdots \cdots, x_{2 n-1}^{6}(t) x_{2 n}^{2}(t)$ are higher order infinity as $x_{i}(t)(1 \leq i \leq n)$ tend to infinity. Since $0<\varepsilon_{2 i} l_{2 i}(1 \leq i \leq n)$, so there exists a sufficiently large $L>0$ such that

$\left.V^{\prime}(t)\right|_{(8)}<0$ as $\left|x_{i}\right|>L(1 \leq i \leq n)$, implying that all solutions of system $(8)$ are bounded.

\section{Main Result}

First we discuss the stability of the trivial solution of system (8) (or (9)). Noting that $g(X)$ is a higher order infinitesimal in a neighborhood of $\|X\|=0$. Therefore, the stability of trivial solution of system (10) guarantees the stability of trivial solution of system (8). We consider the following auxiliary system:

$$
X^{\prime}(t)=A X(\mathrm{t})+B X\left(t-\tau^{*}\right)
$$

where $\quad \tau^{*}=\max \left\{\tau_{1}, \tau_{3}, \cdots, \tau_{2 n-1}\right\} \quad, \quad X\left(t-\tau^{*}\right)=$ $\left[x_{1}\left(t-\tau^{*}\right), 0, x_{3}\left(t-\tau^{*}\right), 0, \cdots, x_{2 n-1}\left(t-\tau^{*}\right), 0\right]^{T}$.

Theorem 1 Assume that system (8) has a unique equilibrium point, for selected parameter values

of $a_{2 i}, b_{2 i-1}, c_{2 i-1}$, and $\varepsilon_{2 i}(1 \leq i \leq n)$. Let the eigenvalues of matrix $\mathrm{R}=A+B$ be $\gamma_{i}(1 \leq i \leq 2 n)$. If $\operatorname{Re}\left(\gamma_{i}\right)(i=$ $1,2, \cdots, 2 n)<-r<0$, then the unique equilibrium point, namely, the trivial solution of system (8) is stable.

Proof Since $\operatorname{Re}\left(\gamma_{i}\right)(i=1,2, \cdots, 2 n)<-r<0$, hence there exists a positive constant $K \geq 1$ such that $\left\|e^{(A+B) t}\right\| \leq$ $K e^{-r t}$. In (15) for $t \geq \tau^{*}$ we have

$$
\begin{gathered}
X^{\prime}(\mathrm{t})=(A+B) X(\mathrm{t})-B \int_{t-\tau^{*}}^{t} X^{\prime}(s) d s \\
=(A+B) X(\mathrm{t})-B \int_{t-\tau^{*}}^{t}\left(A X(s)+B\left(s-t a u^{*}\right)\right) d s
\end{gathered}
$$

From (16), for $t \geq \tau^{*}$ we get

$$
\begin{gathered}
X(t)=e^{(A+B)\left(t-\tau^{*}\right)} X\left(\tau^{*}\right)- \\
B \int_{\tau^{*}}^{t} d s \int_{s-\tau^{*}}^{s} e^{(A+B)(t-s)}\left(\mathrm{AX}(\mathrm{u})+\mathrm{B}\left(\mathrm{u}-t a u^{*}\right)\right) \mathrm{du}
\end{gathered}
$$

Therefore,

$$
\begin{array}{r}
\left\|K e^{-r\left(t-\tau^{*}\right)}+K\right\| B \| \int_{\tau^{*}}^{t} d s \int_{s-\tau^{*}}^{s} e^{-r(t-s)}(\|A\|\|X(u)\| \leq \\
\left.\|B\|\left\|X\left(u-\tau^{*}\right)\right\|\right) d u
\end{array}
$$

where $L=\sup _{t \in\left[-\tau^{*}, \tau^{*}\right]}\|X(t)\|$. We shall prove that there exists a positive constant $c(<r)$ such that $\|X(t)\| \leq$ $L K e^{-c\left(t-\tau^{*}\right)}, t \geq \tau^{*}$. Indeed, select $c(<r)$ such that

$$
K\|B\|\left(\|A\|+\|B\| e^{c \tau^{*}}\right)\left(e^{c \tau^{*}}-1\right)=c(r-c)
$$

then from (18) we have

$$
\begin{aligned}
\|X(t)\| & \leq L K e^{-r\left(t-\tau^{*}\right)}+K\|B\| \int_{\tau^{*}}^{t} d s \int_{s-\tau^{*}}^{s} e^{-r(t-s)}\left(\|A\| L K e^{-c\left(u-\tau^{*}\right)}+\|B\| L K e^{-c\left(u-2 \tau^{*}\right)}\right) d u \\
= & L K e^{-r\left(t-\tau^{*}\right)}+K\|B\| \frac{c K\left(\|A\| e^{c \tau^{*}}+\|B\| e^{2 c \tau^{*}}\right)}{-c} \int_{\tau^{*}}^{t} e^{-r(t-s)}\left(e^{-c s}-e^{-c\left(s-\tau^{*}\right)}\right) d s \\
& =L K e^{-r\left(t-\tau^{*}\right)}+K\|B\| \frac{c K\left(\|A\|+\|B\| e^{c \tau^{*}}\right)\left(e^{c \tau^{*}}-1\right)}{-c(r-c)}\left(e^{-c\left(s-\tau^{*}\right)}-e^{-r\left(t-\tau^{*}\right)}\right) \\
& =L K e^{-c\left(t-\tau^{*}\right)}
\end{aligned}
$$

This means that $X(t) \rightarrow 0$ as $t \rightarrow \infty$ in system (15). Since $\tau_{2 i-1} \leq \tau^{*}(i=1,2, \cdots, n)$, and $g(X)$ is higher infinitesimal as $X(t) \rightarrow 0$, based on the property of delayed differential equation, we know that the trivial solution of system (8) (or (9)) is stable. The proof is completed.

Theorem 2 Assume that system (8) has a unique equilibrium point, for selected parameter values of $a_{2 i}, b_{2 i-1}, c_{2 i-1}$, and $\varepsilon_{2 i}(1 \leq i \leq n)$. Let the eigenvalues of matrix $\mathrm{R}=A+B$ be $\gamma_{i}(1 \leq i \leq 2 n)$, the eigenvalues of matrix $A$ be $\rho_{i}(1 \leq i \leq 2 n)$. If there exists at least one eigenvalue $\rho_{k}, k \in\{1,2, \cdots, 2 n\}$ such that $\operatorname{Re}\left(\rho_{k}\right)>0$, then the unique equilibrium point of system (8) is unstable, implying that system (8) generates an oscillatory solution.
Proof Obviously, if the trivial solution of system (10) is unstable, then the trivial solution of system (9) is also unstable. Therefore, we only need to consider the stability of the trivial solution of system (10). The characteristic equation corresponding to system (10) is

$$
\operatorname{det}\left(\lambda I_{i j}-a_{i j}-b_{i j} e^{-\lambda \tau_{j}}\right)=0
$$

Noting that each characteristic value of matrix $B$ is zero. So we have

$$
\operatorname{det}\left(\lambda I_{i j}-a_{i j}-b_{i j} e^{-\lambda \tau_{j}}\right)=\prod_{i=1}^{2 n} \lambda_{i}-\rho_{i}=0
$$

By the assumption, there exits at least one $k$ such that $\mathrm{Re}$ 
$\left(\lambda_{\mathrm{k}}\right)=\operatorname{Re}\left(\rho_{k}\right)>0$, this means that the trivial solution of system (10) is unstable, implying that the trivial solution of system (8) (or (9)) is unstable. Since all solutions of system (8) (or (9)) are bounded, and system (8) has a unique unstable equilibrium. Based on the generalized Chafee's criterion, this instability of the unique equilibrium will force system (8) to generate an oscillatory solution.

Theorem 3 Assume that system (8) has a unique equilibrium point, for selected parameter values of $a_{2 i}, b_{2 i-1}, c_{2 i-1}$, and $\varepsilon_{2 i}(1 \leq i \leq n)$. If there exists one $\varepsilon_{2 k} a_{2 k}, k \in\{1,2, \cdots, n\}$ such that

$$
c_{2 k-1}-\varepsilon_{2 k} a_{2 k}<0
$$

then the unique equilibrium point of system (10) is unstable, implying that system (8) generates an oscillatory solution.

Proof As theorem 2, we only need to consider the instability of the trivial solution of system (10). For some $k \in\{1,2, \cdots, n\}$, consider an auxiliary equation

$$
\begin{gathered}
y_{2 k}^{\prime}(t)=-c_{2 k-1} y_{2 k}(t)+b_{2 k}\left[y_{2 k}\left(t-\tau_{2 k-3}\right)\right. \\
-2 y_{2 k}\left(t-\tau_{2 k-1}\right) \\
\left.+y_{2 k}\left(t-\tau_{2 k+1}\right)\right]+\varepsilon_{2 k} a_{2 k} y_{2 k}(t)
\end{gathered}
$$

The characteristic equation of (23) is the following

$$
\begin{aligned}
& \lambda+c_{2 k-1}-\varepsilon_{2 k} a_{2 k}-b_{2 k} e^{-\lambda \tau_{2 k-3}}+2 b_{2 k} e^{-\lambda \tau_{2 k-1}}- \\
& \left\{\begin{array}{c}
x_{1}^{\prime}(t)=x_{2}(t), \\
\left.x_{2}^{\prime}(t)=-c_{1} x_{1}(t)-\beta_{1} x_{1}^{3}(t)+b_{1}\left[x_{5}\left(t-\tau_{5}\right)-2 x_{1} t-\tau_{1}\right)+x_{3}\left(t-\tau_{3}\right)\right] \\
+\varepsilon_{2} a_{2} x_{2}(t)-\varepsilon_{2} x_{1}^{2}(t) x_{2}(t)+\varepsilon_{2} k_{2} x_{1}^{4}(t) x_{2}(t)-\varepsilon_{2} l_{2} x_{1}^{6}(t) x_{2}(t), \\
x_{3}^{\prime}(t)=x_{4}(t), \\
x_{4}^{\prime}(t)=-c_{3} x_{3}(t)-\beta_{3} x_{3}^{3}(t)+b_{3}\left[x_{1}\left(t-\tau_{1}\right)-2 x_{3}\left(t-\tau_{3}\right)+x_{5}\left(t-\tau_{5}\right)\right] \\
+\varepsilon_{4} a_{4} x_{4}(t)-\varepsilon_{4} x_{3}^{2}(t) x_{4}(t)+\varepsilon_{4} k_{4} x_{3}^{4}(t) x_{4}(t)-\varepsilon_{4} l_{4} x_{3}^{6}(t) x_{4}(t), \\
x_{5}^{\prime}(t)=x_{6}(t), \\
x_{6}^{\prime}(t)=-c_{5} x_{5}(t)-\beta_{5} x_{5}^{3}(t)+b_{5}\left[x_{3}\left(t-\tau_{3}\right)-2 x_{5}\left(t-\tau_{5}\right)+x_{1}\left(t-\tau_{1}\right)\right] \\
+\varepsilon_{6} a_{6} x_{6}(t)-\varepsilon_{6} x_{5}^{2}(t) x_{6}(t)+\varepsilon_{6} k_{6} x_{5}^{4}(t) x_{6}(t)-\varepsilon_{6} l_{6} x_{5}^{6}(t) x_{6}(t) .
\end{array}\right.
\end{aligned}
$$

The parameter values are selected as $c_{1}=5.45, c_{3}=5.65$, $c_{5}=5.85 ; \quad b_{1}=0.075, \quad b_{3}=0.085, b_{5}=0.095 ; a_{2}=$ $-1.05, a_{4}=-0.55, a_{6}=-0.75 ; \quad \beta_{1}=0.35, \quad \beta_{3}=0.25$, $\beta_{5}=0.45 ; l_{2}=0.45, l_{4}=0.25, l_{6}=0.38 ; \varepsilon_{2}=0.0005$, $\varepsilon_{4}=0.0002, \varepsilon_{6}=0.0004, \quad$ and $\quad k_{2}=0.35, k_{4}=0.42$, $k_{6}=0.68$, respectively, the eigenvalues of matrix $R_{1}=A_{1}+$ $B_{1}$ are $-0.0002 \pm 7.5168 i, \quad-0.0002 \pm 2.4001 i$, and $-0.0002 \pm 0.7792 i$. Obviously, the conditions of Theorem 1 are satisfied. The solutions of system (26) are convergent (see Figure 1). When the parameter values are selected as $c_{1}=5.12, \quad c_{3}=5.15, \quad c_{5}=5.18 ; \quad b_{1}=0.00175, \quad b_{3}=$ $0.00185, b_{5}=0.00165 ; a_{2}=0.95, a_{4}=0.55, a_{6}=0.75$; $\beta_{1}=0.25, \quad \beta_{3}=0.15, \quad \beta_{5}=0.45 ; \quad l_{2}=0.15, \quad l_{4}=0.24$, $l_{6}=0.18 ; \quad \varepsilon_{2}=0.0005, \quad \varepsilon_{4}=0.0002, \varepsilon_{6}=0.0004$, and $k_{2}=0.25, k_{4}=0.32, \quad k_{6}=0.16, \quad$ respectively. The eigenvalues of matrix $A_{1}$ are $0.0024 \pm 2.2803 i, 0.0001 \pm$ $2.2694 i$, and $0.0002 \pm 2.2760 i$, the conditions of Theorem 2 are satisfied. System (26) generates an oscillatory solutions

$$
b_{2 k} e^{-\lambda \tau_{2 k+1}}=0
$$

We show that the characteristic equation (24) of the auxiliary equation has a real positive root say $\lambda^{*}(>0)$. Define a function

$$
\begin{gathered}
h(\lambda)=\lambda+c_{2 k-1}-\varepsilon_{2 k} a_{2 k}-b_{2 k} e^{-\lambda \tau_{2 k-3}}+ \\
2 b_{2 k} e^{-\lambda \tau_{2 k-1}}-b_{2 k} e^{-\lambda \tau_{2 k+1}}
\end{gathered}
$$

Obviously, $h(\lambda)$ is a continuous function of $\lambda$. Under the restrictive condition (22) we have $h(0)=c_{2 k-1}-\varepsilon_{2 k} a_{2 k}-$ $b_{2 k}+2 b_{2 k}-b_{2 k}=c_{2 k-1}-\varepsilon_{2 k} a_{2 k}<0$. Noting that $e^{-\lambda \tau_{2 k-3}} \rightarrow 0$ as $\lambda \rightarrow \infty, e^{-\lambda \tau_{2 k-1}} \rightarrow 0$ as $\lambda \rightarrow \infty$, and $e^{-\lambda \tau_{2 k+1}} \rightarrow 0$ as $\lambda \rightarrow \infty$. Therefore, there exists a suitably large $\lambda$ say $\lambda_{1}(>0)$ such that $h\left(\lambda_{1}\right)=\lambda_{1}+c_{2 k-1}-$ $\varepsilon_{2 k} a_{2 k}-b_{2 k} e^{-\lambda_{1} \tau_{2 k-3}}+2 b_{2 k} e^{-\lambda_{1} \tau_{2 k-1}}-b_{2 k} e^{-\lambda_{1} \tau_{2 k+1}}>$ 0 . By means of the Intermediate Value Theorem of continuous function, there exists a $\lambda^{*} \in\left(0, \lambda_{1}\right)$ such that $h\left(\lambda^{*}\right)=0$, where $\lambda^{*}$ is a positive characteristic root of equation (25). This means that the trivial solution of the auxiliary equation (23) is unstable, implying that the trivial solution of system (10) is also unstable. Based on the generalized Chafee's criterion, this instability of the unique equilibrium will force system (8) to generate an oscillatory solution.

Example 1 Consider the case of $n=3$ in the following: (see Figure 2). When the parameter values are selected as $c_{1}=25.12, c_{3}=25.15, c_{5}=25.18$, the other parameters are the same as in Figure 2, we see that the oscillation of the solutions is maintained. However, the oscillatory amplitude and frequency both are changed (see Figure 3), implying that the values of $c_{1}, c_{3}$ and $c_{5}$ affect the oscillatory amplitude and frequency very much of the solutions. When the parameter values are selected as $c_{1}=0.002, c_{3}=0.0015, c_{5}=$ $0.0018 ; b_{1}=0.00175, b_{3}=0.00155, b_{5}=0.00165 ; a_{2}=$ $10.95, a_{4}=10.55, a_{6}=10.75 ; \quad \beta_{1}=0.25, \quad \beta_{3}=0.15$, $\beta_{5}=0.45 ; l_{2}=0.15, l_{4}=0.24, l_{6}=0.18 ; \varepsilon_{2}=0.0005$, $\varepsilon_{4}=0.0002, \varepsilon_{6}=0.0004, \quad$ and $\quad k_{2}=0.25, k_{4}=0.32$, $k_{6}=0.16$, respectively, we have $c_{1}-\varepsilon_{2} a_{2}=-0.0035<0$, $c_{3}-\varepsilon_{4} a_{4}=-0.0006<0$, and $c_{5}-\varepsilon_{6} a_{6}=-0.0025<0$. The conditions of Theorem 3 are satisfied. System (26) generates an oscillatory solutions (see Figure 4).

Example 2 Consider the case of $n=4$ in the following: 


$$
\left\{\begin{array}{c}
x_{1}^{\prime}(t)=x_{2}(t), \\
\left.x_{2}^{\prime}(t)=-c_{1} x_{1}(t)-\beta_{1} x_{1}^{3}(t)+b_{1}\left[x_{7}\left(t-\tau_{7}\right)-2 x_{1} t-\tau_{1}\right)+x_{3}\left(t-\tau_{3}\right)\right] \\
+\varepsilon_{2} a_{2} x_{2}(t)-\varepsilon_{2} x_{1}^{2}(t) x_{2}(t)+\varepsilon_{2} k_{2} x_{1}^{4}(t) x_{2}(t)-\varepsilon_{2} l_{2} x_{1}^{6}(t) x_{2}(t), \\
x_{3}^{\prime}(t)=x_{4}(t), \\
x_{4}^{\prime}(t)=-c_{3} x_{3}(t)-\beta_{3} x_{3}^{3}(t)+b_{3}\left[x_{1}\left(t-\tau_{1}\right)-2 x_{3}\left(t-\tau_{3}\right)+x_{5}\left(t-\tau_{5}\right)\right] \\
+\varepsilon_{4} a_{4} x_{4}(t)-\varepsilon_{4} x_{3}^{2}(t) x_{4}(t)+\varepsilon_{4} k_{4} x_{3}^{4}(t) x_{4}(t)-\varepsilon_{4} l_{4} x_{3}^{6}(t) x_{4}(t), \\
x_{5}^{\prime}(t)=x_{6}(t), \\
x_{6}^{\prime}(t)=-c_{5} x_{5}(t)-\beta_{5} x_{5}^{3}(t)+b_{5}\left[x_{3}\left(t-\tau_{3}\right)-2 x_{5}\left(t-\tau_{5}\right)+x_{7}\left(t-\tau_{7}\right)\right] \\
+\varepsilon_{6} a_{6} x_{6}(t)-\varepsilon_{6} x_{5}^{2}(t) x_{6}(t)+\varepsilon_{6} k_{6} x_{5}^{4}(t) x_{6}(t)-\varepsilon_{6} l_{6} x_{5}^{6}(t) x_{6}(t), \\
x_{7}^{\prime}(t)=x_{8}(t), \\
x_{8}^{\prime}(t)=-c_{7} x_{7}(t)-\beta_{7} x_{7}^{3}(t)+b_{7}\left[x_{5}\left(t-\tau_{5}\right)-2 x_{7}\left(t-\tau_{7}\right)+x_{1}\left(t-\tau_{1}\right)\right] \\
+\varepsilon_{8} a_{8} x_{8}(t)-\varepsilon_{8} x_{7}^{2}(t) x_{8}(t)+\varepsilon_{8} k_{8} x_{7}^{4}(t) x_{8}(t)-\varepsilon_{8} l_{8} x_{7}^{6}(t) x_{8}(t) .
\end{array}\right.
$$

The parameter values are selected as $c_{1}=0.0025, c_{3}=$ $0.0024, \quad c_{5}=0.0022 c_{7}=0.0028 ; \quad b_{1}=0.00135, \quad b_{3}=$ $0.00125, b_{5}=0.00115, b_{7}=0.00125 ; a_{2}=12.85, a_{4}=$ $12.65, a_{6}=12.75, a_{8}=12.45 ; \quad \beta_{1}=0.28, \quad \beta_{3}=0.16$, $\beta_{5}=0.22, \beta_{7}=0.25 ; l_{2}=0.16, l_{4}=0.24, l_{6}=0.18, l_{8}=$ $0.22 ; \quad \varepsilon_{2}=0.00024, \quad \varepsilon_{4}=0.00035, \varepsilon_{6}=0.00032, \quad \varepsilon_{8}=$ 0.00036 , and $k_{2}=0.35, k_{4}=0.32, k_{6}=0.26, k_{8}=0.36$, respectively. We have $c_{1}-\varepsilon_{2} a_{2}=-0.0005<0, c_{3}-$ $\varepsilon_{4} a_{4}=-0.002<0, \quad c_{5}-\varepsilon_{6} a_{6}=-0.0019<0, \quad$ and $c_{7}-\varepsilon_{8} a_{8}=-0.0019<0$. The conditions of Theorem 3 are satisfied. System (27) generates an oscillatory solutions (see Figures 5, 6 and Figure 7).
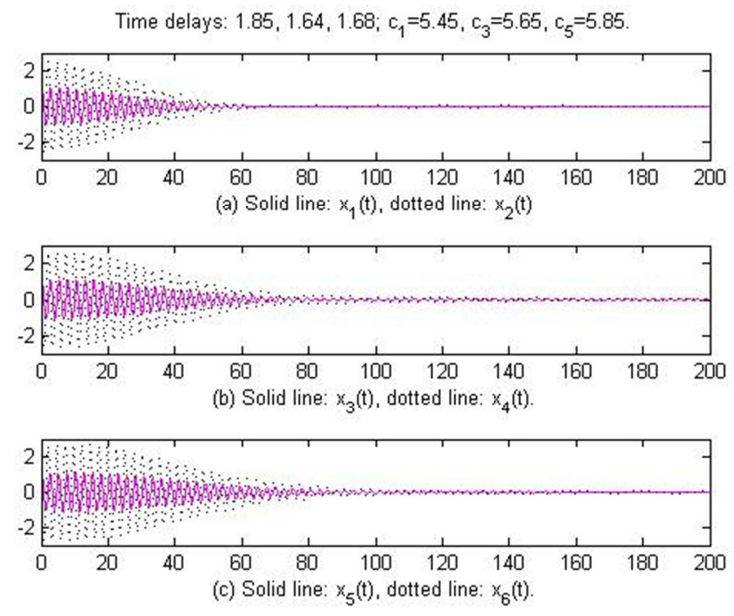

Figure 1. Convergence of the solutions.
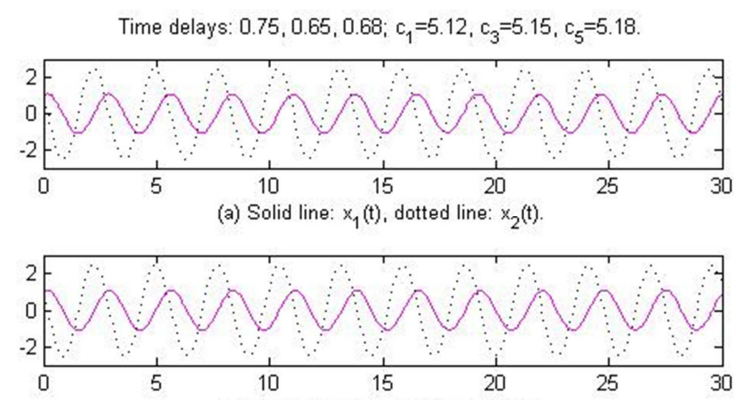

(b) Solid line: $x_{3}(t)$, dotted line: $x_{4}(t)$.

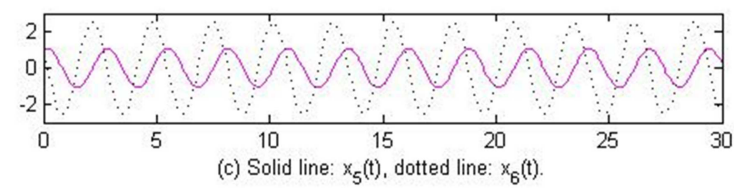

Figure 2. Oscillation of the solutions with $c_{1}=5.12, c_{2}=5.15, c_{3}=5.18$.
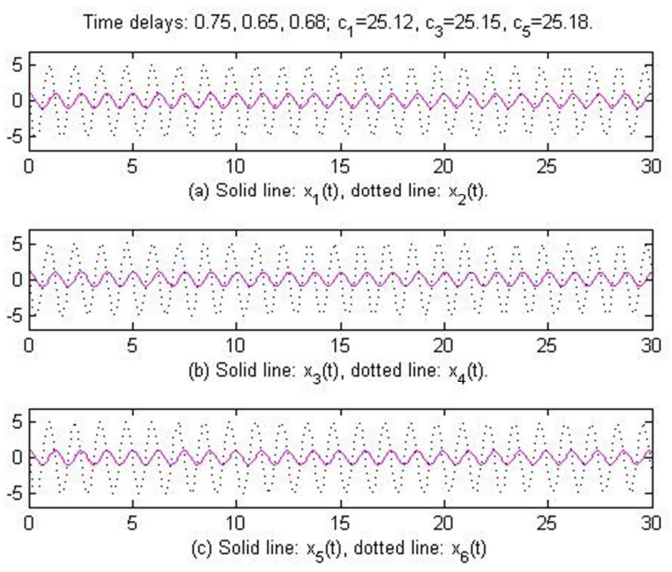

Figure 3. Oscillation of the solutions with $c_{1}=25.12, c_{2}=25.15, c_{3}=25.18$.
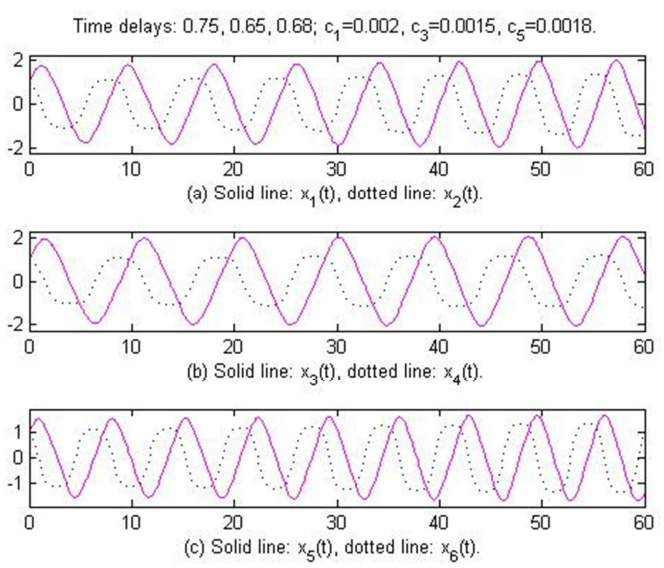

Figure 4. Oscillation of the solutions.
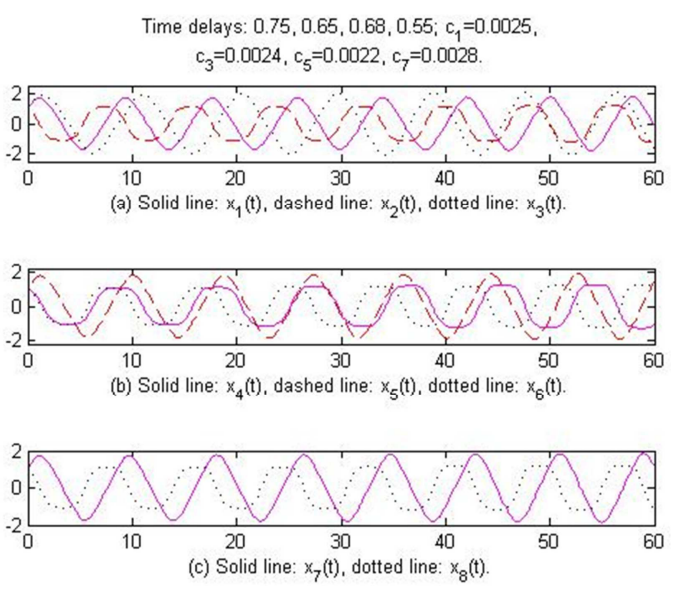

Figure 5. Oscillation of the solutions with delays: $0.75,0.65,0.68,0.55$. 


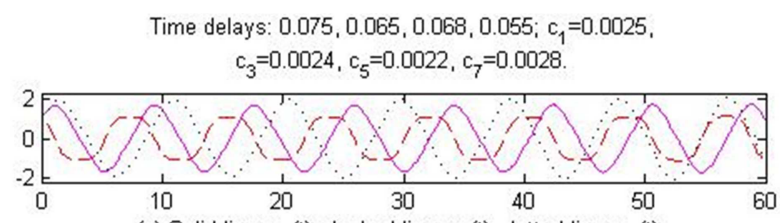

(a) Solid line: $x_{1}(t)$, dashed line: $x_{2}(t)$, dotted line: $x_{3}(t)$.

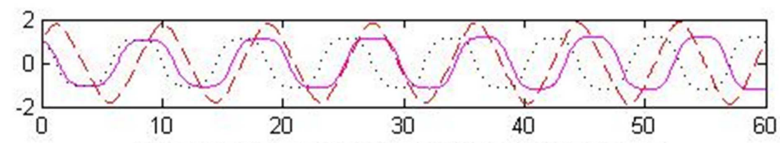

(b) Solid line: $x_{4}(t)$, dashed line: $x_{5}(t)$, dotted line: $x_{6}(t)$.

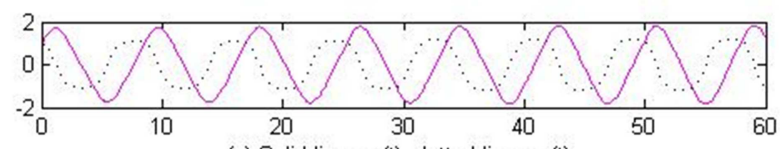

(c) Solid line: $x_{7}(t)$, dotted line: $x_{8}(t)$

Figure 6. Oscillation of the solutions with delays: $0.075,0.065,0.068$, 0.055 .

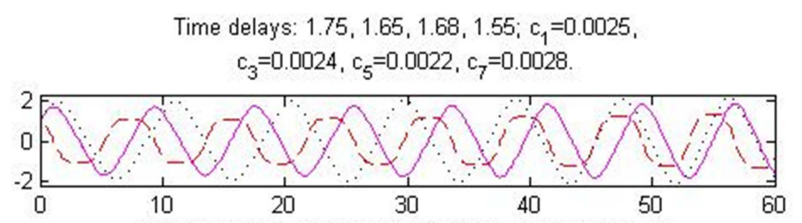

(a) Solid line: $x_{1}(t)$, dashed line: $x_{2}(t)$, dotted line: $x_{3}(t)$.

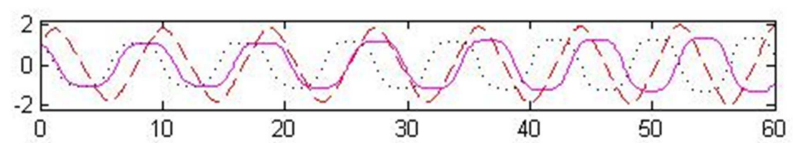

(b) Solid line: $x_{4}(t)$, dashed line: $x_{5}(t)$, dotted line: $x_{6}(t)$.

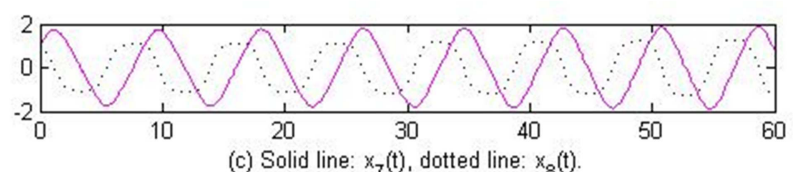

Figure 7. Oscillation of the solutions with delays: $1.75,1.65,1.68,1.55$.

\section{Discussion}

When system generates an oscillatory solution, from the Figures we know that time delay affects the oscillatory amplitude and frequency not too much. However, the positive parameter values $c_{1}, c_{3}, c_{5}, c_{7}$ affect the stability and oscillation of the system. The oscillatory frequency changes too much when different values of $c_{1}, c_{3}, c_{5}$, and $c_{7}$ are selected.

\section{Conclusion}

In this paper, we have discussed the oscillatory behavior of the solutions on a class of coupled van der Pol-Duffing equations with delays. Based on the generalized Chafee's theory, a simple criterion to guarantee the existence of permanent oscillations, which is easy to check, as compared to predicting the regions of bifurcation have been proposed. In this network, the passive decay rate affects the oscillatory frequency and amplitude. When these time delay systems generate a permanent oscillation, the delays affect oscillatory frequency and amplitude slightly.

\section{References}

[1] G. F. Kuiate, S. T. Kingni, V. K. Tamba, and P. K. Talla, Three-dimensional chaotic autonomous van der pol-Duffing type oscillator and its fractional-order form, Chinese J. Physics, 2018, 56, 2560-2573.

[2] M. A. Barron, Stability of a ring of coupled van der Pol oscillators with non-uniform distribution of the coupling parameter, J. Applied Research Technology, 2016, 14 (1), 6266.

[3] P. Kumar, A. Kumar, and S. Erlicher, A modified hybrid Van der Pol-Duffing-Rayleigh oscillator for modelling the lateral walking force on a rigid floor, Physica D, 2017, 358, $1-14$.

[4] S. F. Wen, Y. J. Shen, X. H. Li, and S. P. Yang, Dynamical analysis of Mathieu equation with two kinds of van der Pol fractional-order terms, Inter. J. Nonlinear Mach. 2016, 84 (1), 130-138.

[5] K. Rompala, R. Rand, and H. Howland, Dynamics of three coupled van der Pol oscillators with application to circadian rhythms, Commun Nonlinear Sci Numer Simulat, 2007, 12 (5), 794-803.

[6] H. G. Kadji, J. B. Orou, and P. Woafo, Synchronization dynamics in a ring of four mutually coupled biological systems, Commun Nonlinear Sci Numer Simulat, 2008, 13 (7), 1361-1372.

[7] R. Yamapi, R. M. Yonkeu, G. Filatrella, and C. Tchawoua, Effects of noise correlation on the coherence of a forced van der Pol type birhythmic system, Commun Nonlinear Sci Numer Simulat, 2018, 62, 1-17.

[8] V. K. Chandrasekar, A. Venkatesan, and I. R. Mohamed, Duffing-van der Pol oscillator type dynamics in MuraliLakshmanan-Chua (MLC) circuit, Chaos, Solitons and Fractals, 2016, 82, 60-71.

[9] D. L. Wang, W. Xu, J. W. Xu, X. D. Gu, and G. D. Yang, Resonance responses in a two-degree-of-freedom viscoelastic oscillator under randomly disordered periodic excitations, Commun Nonlinear Sci Numer Simulat, 2019, $68,302-316$.

[10] J. Brechtl, X. Xie, and P. K. Liaw, Investigation of chaos and memory effects in the Bonhoeffer-van der Pol oscillator with a non-ideal capacitor, Commun Nonlinear Sci Numer Simulat, 2019, 73, 195-216.

[11] P. V. Kuptsov, and A. V. Kuptsova, Radial and circular synchronization clusters in extended starlike network of van der Pol oscillators, Commun Nonlinear Sci Numer Simulat, 2017, 50, 115-127.

[12] L. Makouo, and P. Woafo, Experimental observation of bursting patterns in Van der Pol oscillators, Chaos, Solitons and Fractals, 2017, 94, 95-101.

[13] X. Li, J. Ji, and C. H. Hansen, Dynamics of two delay coupled van der Pol oscillators, Mech. Res. Commun. 2006, 33 (5), 614-627. 
[14] J. M. Zhang, and X. S. Gu, Stability and bifurcation analysis in the delay-coupled van der Pol oscillators, Appl. Math. Model. 2010, 34 (9), 2291-2299.

[15] S. Wirkus, and R. Rand, The dynamics of two coupled van der Pol oscillators with delay coupling, Nonlinear Dyn. 2002, 30, 205-221.

[16] W. Y. Wang, and L. J. Chen, Weak and non-resonant double Hopf bifurcations in $m$ coupled van der Pol oscillators with delay coupling, Appl. Math. Model. 2015, 39 (10-11), 30943102 .

[17] Z. Ghouli, M. Hamdi, F. Lakrad, and M. Belhaq, Quasiperiodic energy harvesting in a forced and delayed Duffing harvester device, J. Sound and Vibration, 2017, 407, 27 (1), 271-285.

[18] P. Kumar, S. Narayanan, and S. Gupta, Investigations on the bifurcation of a noisy Duffing-Van der Pol oscillator, Probabilistic Engineering Mechanics, 2016, 45, 70-86.

[19] C. R. Zhang, B. D. Zheng, and L. C. Wang, Multiple Hopf bifurcations of three coupled van der Pol oscillators with delay, Appl. Math. Comput. 2011, 217 (17), 7155-7166.

[20] C. R. Zhang, W. X. Li, and K. Wang, Boundedness for network of stochastic coupled van der Pol oscillators with time-varying delayed coupling, Appl. Math. Model. 2013, 37 (7), 5394-5402.
[21] A. Maccari, Vibration control for the primary resonance of the van der Pol oscillator by a time delay state feedback, Intern. J. Nonlinear Mechan. 2003, 38 (1), 123-131.

[22] D. Ghosh, A. R. Chowdhury, and P. Saha, On the various kinds of synchronization in delayed Duffing-Van der Pol system, Commun Nonlinear Sci Numer Simulat, 2008, 13 (4), 790-803.

[23] J. Xu, and K. W. Chung, Effects of time delayed position feedback on a van der Pol-Duffing oscillator, Physica D: Nonlinear Phenomena, 2003, 180 (1-2), 17-39.

[24] R. Rusinek, A. Weremczuk, K. Kecik, and J. Warminski, Dynamics of a time delayed Duffing oscillator, Intern. J. Nonlinear Mechan. 2014, 65, 98-106.

[25] N. Chafee, A bifurcation problem for a functional differential equation of finitely retarded type, J. Math. Anal. Appl. 1971, $35,312-348$.

[26] C. Feng, and R. Plamondon, An oscillatory criterion for a time delayed neural ring network model, Neural Networks, 2012, $29,70-79$. 